Interfaces and Free Boundaries 15 (2013), 477-499

DOI 10.4171/IFB/311

\title{
Optimal regularity for the parabolic no-sign obstacle type problem
}

\author{
JOHN ANDERSSON \\ Department of Mathematics, KTH Royal Institute of Technology, 10044 Stockholm, Sweden \\ E-mail:johnan@kth.se \\ ERIK LINDGREN \\ Department of Mathematics, KTH Royal Institute of Technology, 10044 Stockholm, Sweden \\ E-mail:eriklin@kth.se \\ HENRIK SHAHGHOLIAN \\ Department of Mathematics, KTH Royal Institute of Technology, 10044 Stockholm, Sweden \\ E-mail:henriksh@math.kth.se
}

[Received 26 August 2013 and in revised form 27 September 2013]

We study the parabolic free boundary problem of obstacle type

$$
\Delta u-\frac{\partial u}{\partial t}=f \chi\{u \neq 0\}
$$

Under the condition that $f=H v$ for some function $v$ with bounded second order spatial derivatives and bounded first order time derivative, we establish the same regularity for the solution $u$. Both the regularity and the assumptions are optimal.

Using this result and assuming that $f$ is Dini continuous, we prove that the free boundary is, near so called low energy points, a $C^{1}$ graph.

Our result completes the theory for this type of problems for the heat operator.

2010 Mathematics Subject Classification: Primary 35R35.

Keywords: Free boundary problem, parabolic equation, regularity, Dini condition

\section{Introduction}

We present a proof of the interior optimal regularity, that is, $W_{\infty}^{2,1}$-regularity (bounded second order spatial derivatives and bounded first order time derivative) for solutions to the so-called parabolic no-sign obstacle type problem

$$
\begin{cases}H u:=\Delta u-\frac{\partial u}{\partial t}=f(x, t) \chi\{u \neq 0\} & \text { in } Q_{1}^{-} \\ u=g & \text { on } \partial_{p} Q_{1}^{-}\end{cases}
$$

under minimal assumptions on the data. Here $B_{r}$ is the unit ball, $Q_{r}^{-}=B_{r} \times\left(-r^{2}, 0\right], f=H v$ where $v \in W_{\infty}^{2,1}$. Since we are interested in the interior regularity, the assumptions on $g$ are not very important but we assume that $g$ is bounded and continuous for the sake of definiteness.

Naturally, we cannot expect that a solution $u$ of (1) is in any better regularity class than the solution, $v$, of the heat equation with right hand side $f$

$$
H v=f \text {. }
$$

(C) European Mathematical Society 2013 
Noticing that if $f \in L^{p}$ then $f \chi_{\{u \neq 0\}} \in L^{p}$ so for $f$ in the $L^{p}$-range Calderon-Zygmund theory directly implies that $u \in W_{p}^{2,1}$ (cf. Proposition 7.14 in [10]) - which is the same regularity one can expect for $v$.

It is also easy to see that $u$ is no better than $C^{1,1}$-regular (bounded second order spatial derivatives) in the space directions even in the case when $f$ is constant. As a matter of fact, with an appropriate choice of $g$ the function

$$
u(x, t)=\frac{1}{2}\left(\max \left(0, x_{1}\right)\right)^{2}
$$

is a solution with $f(x, t)=1$. Similarly, the example

$$
u(x, t)=-(t-1 / 2)^{+}
$$

shows that $u(x, t)$ is no better than $C^{0,1}$ regular (bounded first order time derivative) in time. This shows that even for constant $f$ we cannot hope for better regularity than $u \in W_{\infty}^{2,1}$. This leads to the question: What is the weakest possible assumptions on $f$ that assures that a solution $u(x, t)$ of (1) satisfies $u \in W_{\infty}^{2,1}$ ?

Since, in general, a solution to (1) is never more regular than a solution to $H v=f$ one might ask: If $f$ is such that the solution $v$ to $H v=f$ satisfies $v \in W_{\infty}^{2,1}$ will a solution to (1) also satisfy $u \in W_{\infty}^{2,1}$ ?

In the main theorem of this paper we answer the question in the affirmative. This clearly provides an optimal regularity result for the parabolic no-sign obstacle type problem. Our main theorem is the following.

THEOREM 1 Let $u$ be a solution to (1) and assume furthermore that $f=H v$ in $Q_{1}^{-}$where $v \in$ $W_{\infty}^{2,1}\left(Q_{1}^{-}\right)$and that $u \in L^{1}\left(Q_{1}^{-}\right)$. Then $u \in W_{\infty}^{2,1}\left(Q_{1 / 2}^{-}\right)$and

$$
\left\|D^{2} u\right\|_{L^{\infty}\left(Q_{1 / 2}^{-}\right)}+\left\|u_{t}\right\|_{L^{\infty}\left(Q_{1 / 2}^{-}\right)} \leqslant C\left(\|u\|_{L^{1}\left(Q_{1}^{-}\right)}+\left\|D^{2} v\right\|_{L^{\infty}\left(Q_{1}^{-}\right)}+\left\|v_{t}\right\|_{L^{\infty}\left(Q_{1}^{-}\right)}\right),
$$

where $C$ depends on the dimension.

It might be in order to comment on the assumption that $H v=f$ where $v \in W_{\infty}^{2,1}\left(Q_{1}^{-}\right)$. The assumption might look technical, but to the authors' knowledge there is no necessary condition on $f$ that assures that the solution $v$ to $H v=f$ is in $W_{\infty}^{2,1}$. The weakest sufficient condition known to the authors is $f \in C^{\text {Dini }}\left(Q_{1}^{-}\right)$(see Section 1.1 and for instance [13] or [16]). Naturally, the theorem holds, with minor changes in (2), under the assumption that $f \in C^{\text {Dini }}\left(Q_{1}^{-}\right)$. But the assumption we make on $f$ is strictly weaker than Dini-continuity and it also highlights that up to $W_{\infty}^{2,1}$ the regularity of solutions to the parabolic no-sign obstacle type problem are as regular as the solutions to the corresponding heat equation.

Before we give a brief sketch of the history of the problem and formulate our second main theorem we would like to remark that this is a free boundary result. As a matter of fact the problem can be formulated as follows. Given $f \in L^{p}\left(Q_{1}^{-}\right)$and $g \in C\left(\partial_{p} Q_{1}^{-}\right)$find a set $\Omega \subset Q_{1}^{-}$and a function $u \in W_{p}^{2,1}\left(Q_{1}^{-}\right)$solving

$$
\begin{cases}H u=f \chi_{\Omega} & \text { in } Q_{1}^{-}, \\ u=|\nabla u|=0 & \text { in } Q_{1}^{-} \backslash \Omega, \\ u=g & \text { on } \partial_{p} Q_{1}^{-} .\end{cases}
$$


The free boundary is $\partial_{p}$ interior $(\{u=0\}) \cap Q_{1}^{-}=\partial_{p}\{u \neq 0\} \cap Q_{1}^{-}$. In our proof we will use that $u=0$ outside of $\operatorname{spt}(H u)$ (to be more precise, we use that $\left\|D^{2} u\right\|=u_{t}=0$ a.e.). The above theorem is not true, in general, for solutions to

$$
\begin{cases}H u=f \chi_{\Sigma} & \text { in } Q_{1}^{-}, \\ u=g & \text { on } \partial_{p} Q_{1}^{-},\end{cases}
$$

where $\Sigma$ is an arbitrary set. A simple counterexample for the time independent case, with $\Sigma=$ $\left\{x_{1} x_{2}>0\right\}$, is given in [2]. In [2] a solution, $u$, to (3) with $f(x, t)=-1$ and $\Sigma=\left\{x_{1} x_{2}>0\right\}$ is explicitly calculated and $u(x, t)=u(x, 0) \neq C^{1,1}\left(B_{1}(0)\right)$. That $f(x, t)<0$ in [2], whereas we usually think of $f>0$ in obstacle problems is irrelevant since (3) is linear, so we can simply change the sign of the equation by taking $-u$ instead of $u$. The moral sense of the example is that the solution to the free boundary problem choses a zero level set that assures that the solution has slightly better regularity than what one in general would expect a solution to the similar problem (3) to have.

Before we continue we would like to sketch an outline of some previous research into this problem. The aim of this outline is not to provide a historical survey but to situate our result in the current theory for obstacle type problems.

The papers we discuss below ( [4], [8], [14] [7]) are technically very sophisticated and we have to refer the reader to the original sources for the full details. It should be mentioned that we will, rather mischievously, slightly change the conceptual framework of the above papers into the BMO framework of this paper in our explanations.

If $f \in L^{\infty}\left(Q_{1}^{-}\right)$then it directly follows that $\tilde{D}^{2} u \in B M O\left(Q_{1 / 2}^{-}\right)$, i.e.,

$$
\left\|\tilde{D}^{2} u-\left(\tilde{D}^{2} u\right)_{Q_{r}^{-}\left(X^{0}\right)}\right\|_{L^{2}\left(Q_{r}^{-}\left(X^{0}\right)\right)} \leqslant C r^{(n+2) / 2}\left(\|u\|_{L^{1}\left(Q_{1}^{-}\right)}+\|f\|_{L^{\infty}\left(Q_{1}^{-}\right)}\right),
$$

where $\left(\tilde{D}^{2} u\right)_{Q_{r}^{-}\left(X^{0}\right)}$ denotes the average over the parabolic half cylinder $Q_{r}^{-}\left(X^{0}\right)$ and $\tilde{D}^{2}$ is the second spatial and first time derivative (see the list of notation at the end of the introduction). If $\left|\left(\tilde{D}^{2} u\right)_{Q_{r}^{-}\left(X^{0}\right)}\right| \leqslant C$ for some constant $C$ independent of $r$ and $X^{0}$ then by the triangle inequality $\left\|\tilde{D}^{2} u\right\|_{L^{2}\left(Q_{r}^{-}\left(X^{0}\right)\right)} \leqslant C r^{(n+2) / 2}$ which implies that $\tilde{D}^{2} u \in L^{\infty}$. It is not difficult to see (cf. Lemma 12) that instead of subtracting $\left(\tilde{D}^{2} u\right)_{Q_{r}^{-}\left(X^{0}\right)}$ in (4) we can use $\tilde{D}^{2} p_{u, r, X^{0}}(x, t)$ where $p_{u, r, X^{0}}(x, t)$ is a parabolic polynomial that is second order homogeneous in $x$ and first order homogeneous in $t$. In particular it is enough to estimate $\left\|p_{u, r, X^{0}}\right\|_{L^{\infty}\left(Q_{1}^{-}\right)}$in order to derive $W_{\infty}^{2,1}$-regularity for $u$.

The first regularity results for parabolic obstacle type problems were obtained under the assumption that $u \geqslant 0$, which implies that $p_{u, r, X^{0}} \geqslant-C$ in $Q_{1}^{-}$since $\left\|u-p_{u, r, X^{0}}\right\|_{L^{2}\left(Q_{r}^{-}\left(X^{0}\right)\right)} \leqslant$ $C r^{2+(n+2) / 2}$, by our BMO estimate. But if $p_{u, r, X^{0}} \geqslant-C$ and is a second order caloric polynomial then it directly follows that $p_{u, r, X^{0}} \leqslant C$ in $Q_{1}^{-}$which implies the optimal regularity by the above. The real difficulties therefore occur for no-sign obstacle type problems, i.e., when there is no assumption on the sign of the solution.

The first major breakthrough in the regularity theory for parabolic obstacle problems without a sign assumption was achieved in [4], where it was proved that if $f(x, t)=1$ then the solution to (1) is in $W_{\infty}^{2,1}$. The proof is based on a monotonicity formula first proved in [5]. The monotonicity formula is applied on the positive and negative parts of $D_{e} u$, the directional derivatives of $u$, which can be shown to be sub-caloric functions in their supports. The monotonicity formula gives uniform 
bounds, in $r$, of the following averages

$$
\frac{1}{r^{4}} \int_{-r^{2}}^{0} \int_{B_{r}}\left|\nabla\left(D_{e} u\right)^{+}\right|^{2} G(x,-s) d x d s \int_{-r^{2}}^{0} \int_{B_{r}}\left|\nabla\left(D_{e} u\right)^{-}\right|^{2} G(x,-s) d x d s .
$$

Since, BMO-estimates implies $u=p_{u, r, X^{0}}$ up to an error that is bounded in $W_{\infty}^{2,1}$ it follows that the expression in (5) is bounded with $p_{u, r, X^{0}}$ in place of $u$. Using that $p_{u, r, X^{0}}$ is a parabolic second order polynomial implies, by elementary calculations, that $p_{u, r, X^{0}}$ is bounded.

Since the monotonicity formula in [5] is valid only if $\left(D_{e} u\right)^{ \pm}$are sub-caloric functions the results in [4] are only valid in the case when $f$ is constant. However, a refined version of the monotonicity formula, valid when $H\left(D_{e} u\right)^{ \pm} \geqslant-C$ for some constant $C$, was proved in [8]. This monotonicity formula makes it possible to prove $W_{\infty}^{2,1}$-regularity for solutions when $f \in C^{0,1}$. Clearly, if $f \notin C^{0,1}$ then $H\left(D_{e} u\right)^{ \pm} \geqslant-C$ is no longer true which makes it difficult to use this method to prove regularity for $f$ less regular than Lipschitz.

The monotonicity formula approach therefore provides optimal regularity results for $f \in C^{0,1}$. There is however a substantial and rather unsatisfying gap in the regularity theory. If $f \in L^{\infty}$, then classical methods implies that $u \in W_{p}^{2,1}$ for any $p<\infty$. But in order to achieve the $W_{\infty}^{2,1}$-regularity with the above mentioned methods, one needs to assume that $\nabla f \in L^{\infty}$, i.e., a whole extra derivative is required.

There is another approach to the regularity for the parabolic no-sign obstacle type problem, which is based on an extra assumption on the behaviour of the free boundary. It is not difficult to prove that the solution is $W_{\infty}^{2,1}$ close to points $X^{0}$ where $\left|\{u=0\} \cap Q_{r}^{-}\left(X^{0}\right)\right|>\epsilon\left|Q_{r}^{-}\left(X^{0}\right)\right|$ for every $r>0$. The most sophisticated result of this kind is [14] in the elliptic case and [7] for the parabolic case. The assumptions on the free boundary are, in order to be as week as possible, rather technical so we will have to refer the readers to the original papers for the details. For our purposes it is enough to remark that even though the methods in [14] and [7] are strong enough to prove optimal regularity of the solution - they are only able to do so under assumptions on the free boundary which are unfortunately not verifiable in general.

The proof in our paper is based on the method in [1] where we prove similar results for the elliptic problem. Let us sketch the proof of Theorem 1 before we state the second main theorem of the paper. If $u$ is a solution to (1) then we can write

$$
u=v+g+\text { second order caloric polynomial, }
$$

where $g$ is a solution to $H g=-f \chi_{\{u=0\}}$. We will slightly change the notation and write the second order caloric polynomial as $S\left(u, r, X^{0}\right) p_{u, r, X^{0}}$ where $\sup _{Q_{1}^{-}}\left|p_{u, r, X^{0}}\right|=1$, that is, $S\left(u, r, X^{0}\right)$ controls the norm of the caloric polynomial. We will choose $S\left(u, r, X^{0}\right) p_{u, r, X^{0}}$ by means of a projection operator (see Definition 10) that assures that $S\left(u, r, X^{0}\right) p_{u, r, X^{0}}$ closely approximates $u$. As before, the BMO estimates implies that $u \in W_{\infty}^{2,1}$ if and only if $S\left(u, r, X^{0}\right)$ is bounded.

The idea of the proof is to use that on $\Lambda=\{u=0\}$ we have $\tilde{D}^{2} u=0$ and thus

$$
\begin{gathered}
0=\left\|\tilde{D}^{2} u\right\|_{L^{2}\left(\Lambda \cap Q_{r}^{-}\left(X^{0}\right)\right)} \geqslant\left|S\left(u, r, X^{0}\right)\right|\left\|\tilde{D}^{2} p_{u, r, X^{0}}\right\|_{L^{2}\left(\Lambda \cap Q_{r}^{-}\left(X^{0}\right)\right)} \\
-\left\|\tilde{D}^{2} g\right\|_{L^{2}\left(\Lambda \cap Q_{r}^{-}\left(X^{0}\right)\right)}-\left\|\tilde{D}^{2} v\right\|_{L^{2}\left(\Lambda \cap Q_{r}^{-}\left(X^{0}\right)\right) .}
\end{gathered}
$$

Now, since $v \in W_{\infty}^{2,1}$ and since $\left\|p_{u, r, X^{0}}\right\|_{L^{\infty}\left(Q_{1}^{-}\right)}=1$, it follows that

$$
\left\|\tilde{D}^{2} p_{u, r, X^{0}}\right\|_{L^{2}\left(\Lambda \cap Q_{r}^{-}\left(X^{0}\right)\right)} \leqslant \sqrt{\left|\Lambda \cap Q_{r}^{-}\left(X^{0}\right)\right|}
$$


and

$$
\left\|\tilde{D}^{2} v\right\|_{L^{2}\left(\Lambda \cap Q_{r}^{-}\left(X^{0}\right)\right)} \leqslant \sqrt{\left|\Lambda \cap Q_{r}^{-}\left(X^{0}\right)\right|}\left\|\tilde{D}^{2} v\right\|_{L^{\infty}} .
$$

Moreover, $g$ may be written $g=\hat{g}+h$, where $h$ is a caloric function satisfying, by BMO estimates

$$
\left\|\tilde{D}^{2} h\right\|_{L^{2}\left(Q_{r}^{-}\left(X^{0}\right)\right)} \leqslant C r^{(n+2) / 2}\left(\|u\|_{L^{1}\left(Q_{1}^{-}\right)}+\|f\|_{L^{\infty}\left(Q_{1}^{-}\right)}\right),
$$

and $\hat{g}$ has zero boundary data and thus, by parabolic estimates

$$
\left\|\tilde{D}^{2} \hat{g}\right\|_{L^{2}\left(Q_{r}^{-}\left(X_{0}\right)\right.} \leqslant C\|H \hat{g}\|_{L^{2}\left(Q_{r}^{-}\left(X^{0}\right)\right)} \leqslant C \sqrt{\left|\Lambda \cap Q_{r}^{-}\left(X^{0}\right)\right|}\|f\|_{L^{\infty}\left(Q_{1}^{-}\right)} .
$$

If we disregard the caloric function $h$ then (6) can be written as

$$
\left|S\left(u, r, X^{0}\right)\right| \leqslant C\|f\|_{L^{\infty}\left(Q_{1}^{-}\right)},
$$

which is our desired estimate. We may, unfortunately, not disregard $h$ and this explains why the paper is around 20 pages and not just a few lines.

In order to salvage something out of the above calculation we use the parabolic estimate

$$
\left\|\tilde{D}^{2} h\right\|_{L^{\infty}\left(Q_{r / 2}^{-}\left(X_{0}\right)\right)} \leqslant C r^{-(n+2) / 2}\left\|\tilde{D}^{2} h\right\|_{L^{2}\left(Q_{r}^{-}\left(X^{0}\right)\right)},
$$

which means that we can use the calculation (6) in $Q_{r / 2}^{-}\left(X^{0}\right)$. This estimate appears, then applied directly on $g=\hat{g}+h$, in (15) and the calculations following (15) explicates how this revised estimate controls the dyadic decay of the measure $\left|\Lambda \cap Q_{2^{-j}}\right|$. This is the heart of the paper and carried out, with slight variations, in Proposition 14. The dyadic decay of the measure $\left|\Lambda \cap Q_{2^{-j}}\right|$, Proposition 14, implies that if $S\left(u, r, X^{0}\right)$ is large enough then

$$
\frac{\left|\Lambda \cap Q_{r / 2}^{-}\left(X^{0}\right)\right|}{\left|Q_{r / 2}^{-}\left(X^{0}\right)\right|} \leqslant \frac{1}{4} \frac{\left|\Lambda \cap Q_{r}^{-}\left(X^{0}\right)\right|}{\left|Q_{r}^{-}\left(X^{0}\right)\right|},
$$

i.e., the function $\chi_{\Lambda}$ satisfies a Morrey space condition at $X^{0}$ - at least at the scale $r$. This is utilised, in Proposition 15, to show that $g$ is indeed small as long as $S\left(u, r, X^{0}\right)$ is large in comparison to $\|u\|_{L^{1}\left(Q_{1}^{-}\right)}$and $\left\|\tilde{D}^{2} v\right\|_{L^{\infty}\left(Q_{1}^{-}\right)}$. For full details see the main body of the paper.

Our method improves on the previous research. It is strong enough to prove the optimal regularity with minimal assumptions on $f$ and therefore improves on the monotonicity formula approach in [4] or [8] that required $f \in C^{0,1}$. We do not make any assumptions on the solution or its free boundary (such as in [14] or [7]). Furthermore, whereas previous methods have utilised powerful, but rather specialized, monotonicity formulas with limited reach, our method is based on standard $L^{p}$ estimates. This makes it likely that our methods can be extended to cover other equations, such as equations with variable coefficients, higher order equations or fully non-linear equations.

As soon as $W_{\infty}^{2,1}$-regularity have been established we are in the position to apply the powerful free boundary regularity results developed in [4], [7] and [12]. For that we need to assume that $f \in C^{\text {Dini }}$. This assumption is also optimal in the sense that if $f \notin C^{\text {Dini }}$, then there is a time independent solution for which the free boundary is not $C^{1}$ at the origin, but in fact a spiral point, see [3]. We will only sketch the proof to highlight some minor differences, the reader is referred to the original papers for the details. 
If $u$ is a solution to (1) then we define the free boundary, in the usual way, to be

$$
\Gamma(u)=\partial_{p} \text { interior }(\{u=0\}),
$$

where $\partial_{p}$ stands for the parabolic boundary, referring to the points that are not parabolically interior, i.e., the points such that

$$
Q_{r}^{-} \cap(\text { interior }\{u=0\})=\emptyset,
$$

for any $r$ small enough. In this terminology our second main theorem is the following.

THEOREM 2 Let $u$ be a solution to (1) and assume in addition that $f \in C^{\text {Dini }}\left(Q_{1}^{-}\right)$and $f(0,0)=1$.

- If the origin is a low energy point (as in Definition 19) then the free boundary $\Gamma(u)$ (as defined in (7)) is, in a neighbourhood of the origin (which might depend on the solution $u$ ), a (parabolic) $C^{1}$ regular graph.

- There is a modulus of continuity $\sigma$ and $r_{0}>0$ (both depending on $\|u\|_{L^{1}\left(Q_{1}^{-}\right)}$and $\left.\|f\|_{C^{\text {Dini }}\left(Q_{1}^{-}\right)}\right)$) such that if

$$
\frac{\operatorname{MD}\left(\left\{x: u\left(x,-r^{2}\right)=0\right\} \cap B_{r}\right)}{r}>\sigma(r)
$$

for some $r<r_{0}$, then $\Gamma(u)$ is a $C^{1}$ regular graph in $Q_{r / 2}^{-}$. Here MD stands for the minimal diameter.

It is in order to explain the assumptions in Theorem 2 for the non-expert reader. It is well known that the free boundary is not $C^{1}$ everywhere (see [15] for an example in the time independent case) and some extra assumption is needed to exclude that the origin is a singular point of the free boundary. A natural and correct assumption could be that the zero set of $u$ has positive Lebesgue density at the origin, which is a slightly stronger assumption that (8).

There is also a different statement, based on a monotonicity formula, that excludes singularities. The powerful monotonicity formula states that a certain energy $W(r ; u, f,(0,0))$ (defined in Section 6) is almost increasing in $r$ if $u$ is a solution to (1) and $(0,0) \in \Gamma(u)$. Furthermore, $W\left(0^{+} ; u, f,(0,0)\right)$ (which is well defined due to the almost monotonicity) can only assume the values $15,15 / 2$ or 0 , and the value carries geometric information of the free boundary at the origin. For reasons explained in [4], the value 0 in does not occur if $(0,0) \in \Gamma(u)$. If the value is $15 / 2$, we say that the origin is a low energy point.

In particular, by the discreteness of the limiting energies we can conclude that if (8) or a Lebesgue density condition holds then we are at a low energy point. The energy condition we impose is therefore weaker than a Lebesgue density condition but comparable to (8).

\section{$1.1 \quad$ Notation and assumptions}

Throughout the paper we use the following notation: 
$X=(x, t), X^{0}=\left(x^{0}, t^{0}\right), Y=(y, s)$

$u_{t}=\partial_{t} u=\frac{\partial u}{\partial t}$

$\nabla u=\left(\frac{\partial u}{\partial x_{1}}, \ldots, \frac{\partial u}{\partial x_{n}}\right)$

general points in $\mathbb{R}^{n} \times \mathbb{R}^{+}$

$\Delta u=\sum_{i=1}^{n} \frac{\partial^{2} u}{\partial x_{i}{ }^{2}}$

$H u=\Delta u-u_{t}$

$Q_{r}\left(x_{0}, t_{0}\right)=B_{r}\left(x_{0}\right) \times\left(t_{0}-r^{2}, t_{0}+r^{2}\right)$

$Q_{r}^{-}\left(x_{0}, t_{0}\right)=B_{r}\left(x_{0}\right) \times\left(t_{0}-r^{2}, 0\right]$

$Q_{r}=Q_{r}(0,0), \quad Q_{r}^{-}=Q_{r}^{-}(0,0), \quad \partial_{p} Q_{r}^{-}=\partial_{p} Q_{r}^{-}(0,0)$

$W_{p}^{2,1}(A)$

the time derivative

the spatial gradient

the Laplace operator

the heat operator

a parabolic cylinder

a half cylinder

simplified notation

the Sobolev space of functions in

$L^{p}(A)$ with $p$ integrable second

derivatives in space and first

derivatives in time.

$\tilde{D}^{2} u=\left(\begin{array}{lr}D^{2} u & 0 \\ 0 & \partial_{t} u\end{array}\right)$

$\left|\tilde{D}^{2} u\right|^{2}=\sum_{i, j}\left|D_{i, j}^{2} u\right|^{2}+\left|\partial_{t} u\right|^{2}$

the parabolic second derivative

$\Lambda=\{u=0\} \cap Q_{1}^{-}$

$\Gamma=\partial_{p}\left(\{u \neq 0\}^{\circ}\right) \cap Q_{1}^{-}$

$\lambda_{r}=\frac{\left|\Lambda \cap Q_{r}^{-}\right|}{\left|Q_{r}^{-}\right|}$

$(f)_{r, X^{0}}=\frac{1}{\left|Q_{r}^{-}\left(X^{0}\right)\right|} \int_{Q_{r}^{-}\left(X^{0}\right)} f d x d t$

$(f)_{S}=\frac{1}{|S|} \int_{S} f d x d t$

the matrix norm used

the coincidence set

the free boundary, the part of

$\partial\left(\{u \neq 0\}^{\circ}\right)$ that is not

parabolically interior in $\Lambda$

the density of $\Lambda$ at the level $r$

the mean value of $f$ over $Q_{r}^{-}\left(X_{0}\right)$

the mean value of $f$ over

$S \subset \mathbb{R}^{n} \times R$

$(f)_{\Omega}=\frac{1}{\Omega \mid} \int_{\Omega} f d x$

the mean value of $f$ over $\Omega \subset \mathbb{R}^{n}$

$(f)_{(a, b)}=\frac{1}{|b-a|} \int_{a}^{b} f d t$

the mean value of $f$ over the interval $(a, b)$

We will continuously denote by $v$ a function satisfying

$$
v \in W_{\infty}^{2,1}\left(Q_{1}^{-}\right), \quad H v=f \text { in } Q_{1}^{-} .
$$

Finally we introduce the notion of Dini continuity.

DEFINITION 3 (Dini continuity) A function $f(x, t)$ is said to be Dini continuous (in the parabolic setting) if

$$
|f(x, t)-f(y, s)| \leqslant \sigma\left(\sqrt{|x-y|^{2}+|t-s|}\right),
$$

where $\sigma$ is a non-negative continuous function such that $\sigma(0)=0$ and

$$
\int_{0}^{1} \frac{\sigma(s)}{s} d s<\infty .
$$

\section{Parabolic spaces}

Here we present some useful definitions and result for parabolic spaces. 
DEFINITION 4 (Parabolic BMO) We say that a function $f \in L^{2}(\Omega)$ is in $B M O(\Omega)$ if

$$
\|f\|_{B M O(\Omega)}^{2} \equiv \sup _{(x, t) \in \Omega, r>0} \frac{1}{\left|Q_{r}(x, t)\right|} \int_{Q_{r}(x, t) \cap \Omega}\left|f(y, s)-(f) Q_{Q_{r}(x, t)}\right|^{2}+\|f\|_{L^{2}(\Omega)}^{2}<\infty .
$$

The result below is well known and can be found in for instance [6].

THEOREM 5 (BMO-estimates for the heat equation) Let $H w=f$ in $Q_{R}^{-}$. If $f \in L^{\infty}\left(Q_{R}^{-}\right)$then

$$
\left\|D^{2} w\right\|_{B M O\left(Q_{R / 2}^{-}\right)}+\left\|\partial_{t} w\right\|_{B M O\left(Q_{R / 2}^{-}\right)} \leqslant C\left(\|f\|_{L^{\infty}\left(Q_{R}^{-}\right)}+\|w\|_{L^{1}\left(Q_{R}^{-}\right)}\right) .
$$

Here the constant depends only on the space dimension.

We will need the following standard results:

Lemma 6 Let $u$ be a solution of $H u=0$ in $Q_{r}^{-}$. Then

$$
\left\|\tilde{D}^{2} u\right\|_{L^{\infty}\left(Q_{r / 2}^{-}\right)} \leqslant \frac{C}{r^{n+4}}\|u\|_{L^{1}\left(Q_{r}^{-}\right)} .
$$

The proof of this lemma is contained in the proof of Theorem 8 on page 59 in [9].

LEMma 7 Let

$$
\begin{array}{ll}
H u=f & \text { in } Q_{r}^{-} \\
u=0 & \text { on } \partial_{p} Q_{r}^{-}
\end{array}
$$

then

$$
\left\|\tilde{D}^{2} u\right\|_{L^{2}\left(Q_{r}^{-}\right)} \leqslant C\|f\|_{L^{2}\left(Q_{r}^{-}\right)} .
$$

This is a rescaled version of Proposition 7.17 in [10].

LEMMA 8 Let

$$
H w=f \text { in } Q_{1}^{-},
$$

where $f=H v$ for some $v$ satisfying $\left\|\tilde{D}^{2} v\right\|_{L^{\infty}\left(Q_{1}\right)}<\infty$. Then there exists a constant $C$ such that

$$
\left\|\tilde{D}^{2} w\right\|_{L^{\infty}\left(Q_{1 / 2}^{-}\right)} \leqslant C\left(\|w\|_{L^{1}\left(Q_{1}^{-}\right)}+\left\|\tilde{D}^{2} v\right\|_{L^{\infty}\left(Q_{1}^{-}\right)}\right) .
$$

This lemma is an easy consequence of the parabolic version of the Poincaré inequality below.

LEmma 9 (Parabolic Poincaré inequality) Assume that $w \in W_{2}^{2,}\left(Q_{1}^{-}\right)$. Then for some $\kappa \in(7 / 8,1)$ and some $C>0$

$$
\left\|w-(w) Q_{Q^{-}}-x \cdot(\nabla w) Q_{Q_{\kappa}^{-}}\right\|_{L^{2}\left(Q_{\kappa}^{-}\right)} \leqslant C\left(\left\|D^{2} w\right\|_{L^{2}\left(Q_{1}^{-}\right)}+\left\|w_{t}\right\|_{L^{2}\left(Q_{1}^{-}\right)}\right) .
$$

Proof. We notice that from the Poincaré inequality applied of each $t$-section of $w$ we may deduce that

$$
\int_{Q_{\bar{\kappa}}^{-}}\left|w-(w)_{B_{\kappa} \times\{t\}}-x \cdot(\nabla w)_{B_{\kappa} \times\{t\}}\right|^{2} d x d t \leqslant C \int_{Q_{\bar{\kappa}}^{-}}\left|D^{2} w\right|^{2} d x d t .
$$

Hence, it suffices to find proper estimates for the differences

$$
\left\|(w)_{B_{\kappa} \times\{t\}}-(w)_{Q_{k}^{-}}\right\|_{L^{2}\left(Q_{\kappa}^{-}\right)}, \quad\left\|x \cdot(\nabla w)_{B_{\kappa} \times\{t\}}-x \cdot(\nabla w)_{Q_{\kappa}^{-}}\right\|_{L^{2}\left(Q_{\kappa}^{-}\right)} .
$$


For that purpose we first of all remark that from the Poincaré inequality employed for fixed $x$ one can conclude

$$
\int_{Q_{\kappa}^{-}}\left|w-(w)_{\left(-\kappa^{2}, 0\right)}\right|^{2} d x d t \leqslant C \int_{Q_{\kappa}^{-}}\left|w_{t}\right|^{2} d x d t .
$$

From Hölder's inequality we can then conclude

$$
\begin{gathered}
\int_{Q_{\bar{\kappa}}^{-}}\left|(w)_{B_{\kappa} \times\{t\}}-(w)_{Q_{k}^{-}}\right|^{2} d x d t \leqslant \\
\leqslant C \int_{Q_{\bar{\kappa}}}\left(\int_{B_{\kappa}}\left|w-(w)_{\left(-\kappa^{2}, 0\right)}\right|^{2}\right) d x d t \leqslant C \int_{Q_{\bar{\kappa}}^{-}}\left|w_{t}\right|^{2} d x d t .
\end{gathered}
$$

Now we compute, and use for the third equality that $x \cdot \nabla_{y} w(y, \cdot)=\operatorname{div}_{y}(x w(y, \cdot))$ together with the divergence theorem

$$
\begin{aligned}
& \int_{Q_{\bar{\kappa}}^{-}}\left|x \cdot(\nabla w)_{B_{\kappa} \times\{t\}}-x \cdot(\nabla w)_{Q_{\kappa}^{-}}\right|^{2} d x d t \\
& =\int_{Q_{\bar{\kappa}}}\left|x \cdot \frac{1}{\left|B_{\kappa}\right|} \int_{B_{\kappa}} \nabla_{y} w(y, t) d y-x \cdot \frac{1}{\kappa^{2}\left|B_{\kappa}\right|} \int_{Q_{\bar{\kappa}}} \nabla_{y} w(y, s) d y d s\right|^{2} d x d t \\
& =\int_{Q_{\kappa}^{-}}\left|\frac{1}{\left|B_{\kappa}\right|} \int_{\partial B_{\kappa}} x \cdot v w(y, t) d y-\frac{1}{\kappa^{2}\left|B_{\kappa}\right|} \int_{-\kappa^{2}}^{0} \int_{\partial B_{\kappa}} x \cdot v w(y, s) d y d s\right|^{2} d x d t \\
& \leqslant C \int_{Q_{\kappa}^{-}} \int_{\partial B_{\kappa}}\left|w(y, t)-(w(y, s))_{\left(-\kappa^{2}, 0\right)}\right|^{2} d y d x d t \\
& \leqslant C \int_{-\kappa^{2}}^{0} \int_{\partial B_{\kappa}}\left|w(y, t)-(w(y, s))_{\left(-\kappa^{2}, 0\right)}\right|^{2} d t .
\end{aligned}
$$

Using polar coordinates and the mean value theorem, there exist a $\kappa \in(7 / 8,1)$ such that

$$
\begin{aligned}
& \frac{C}{\left|B_{\kappa}\right|} \int_{-\kappa^{2}}^{0} \int_{\partial B_{\kappa}}\left|w(y, t)-(w(y, s))_{\left(-\kappa^{2}, 0\right)}\right|^{2} d y d t \\
& \leqslant C \int_{-1}^{0} \int_{B_{1}}\left|w(y, t)-(w(y, s))_{\left(-\kappa^{2}, 0\right)}\right|^{2} d y d t \\
& \leqslant C \int_{Q_{1}^{-}}\left|w_{t}\right|^{2} d x d t,
\end{aligned}
$$

where the last inequality follows from (11). Hence,

$$
\int_{Q_{k}^{-}}\left|x \cdot(\nabla w)_{B_{\kappa} \times\{t\}}-x \cdot(\nabla w)_{Q_{\kappa}^{-}}\right|^{2} d x d t \leqslant C \int_{Q_{1}^{-}}\left|w_{t}\right|^{2} d x d t .
$$

To conclude the lemma, we only need to combine (10), (11) and (12).

\section{The projection $\Pi$ and some technical results}

DEFINITION 10 Let $\Pi\left(u, r, X^{0}\right)$ be the projection of $u$ into the space of second order parabolic homogeneous caloric polynomials in $Q_{r}^{-}\left(X^{0}\right)$. In other words

$$
\int_{Q_{r}^{-}\left(X^{0}\right)}\left|\tilde{D}^{2} u(X)-\tilde{D}^{2} \Pi\left(u, r, X^{0}\right)\right|^{2}=\inf _{p \in \mathbb{P}_{2}} \int_{Q_{r}^{-}\left(X^{0}\right)}\left|\tilde{D}^{2} u(X)-\tilde{D}^{2} p(X)\right|^{2},
$$


where $P_{2}$ is the space of caloric polynomials that are homogeneous of degree two in space and homogeneous of degree one in time and where we use the matrix norm

$$
|A|^{2}=\sum_{i, j}\left|A_{i, j}\right|^{2}
$$

Moreover, we will use the notation

$$
\Pi\left(u, r, X^{0}\right)=S\left(u, r, X^{0}\right) p_{u, r, X^{0}}(X),
$$

where $p_{u, r, X^{0}}(X)$ is a second order parabolic homogeneous caloric polynomial such that

$$
\left\|\tilde{D}^{2} p_{u, r, X^{0}}\right\|_{L^{\infty}\left(Q_{1}^{-}\right)}=\left|\tilde{D}^{2} p_{u, r, X^{0}}\right|=1
$$

and $S\left(u, r, X^{0}\right) \in \mathbb{R}_{+}$.

Below are certain properties of the projection that can be easily verified.

LEMma 11 Let $u$ be as in Theorem 1 . Then

1. $\Pi\left(\cdot, r, X^{0}\right)$ is linear;

2. $\left\|\Pi\left(u, r, X^{0}\right)\right\|_{L^{2}\left(Q_{1}^{-}\right)} \leqslant C\left\|\tilde{D}^{2} u\right\|_{L^{2}\left(Q_{1}^{-}\right)}$for $r \in\left[\frac{1}{2}, 1\right]$;

In the above, $C$ is a constant depending only on the dimension.

Proof. The first statement follows from the fact that $\Pi$ is a projection.

In order to prove the second statement we observe that if $\tilde{D}^{2} u \in L^{2}\left(Q_{1}^{-}\right)$then

$$
\inf _{P \in \mathbb{P}_{2}} \int_{Q_{1}^{-}}\left|\tilde{D}^{2} u-\tilde{D}^{2} P\right|^{2} \leqslant \int_{Q_{1}^{-}}\left|\tilde{D}^{2} u\right|^{2}
$$

and moreover

$$
\left\|\tilde{D}^{2} u-\tilde{D}^{2} P\right\|_{L^{2}\left(Q_{1}^{-}\right)} \geqslant\left\|\tilde{D}^{2} P\right\|_{L^{2}\left(Q_{1}^{-}\right)}-\left\|\tilde{D}^{2} u\right\|_{L^{2}\left(Q_{1}^{-}\right)} .
$$

Thus,

$$
\left\|\tilde{D}^{2} \Pi(u, 1,0)\right\|_{L^{2}\left(Q_{1}^{-}\right)} \leqslant 2\left\|\tilde{D}^{2} u\right\|_{L^{2}\left(Q_{1}^{-}\right)} .
$$

Since $\tilde{D}^{2} \Pi(u, 1,0)$ is constant and $\Pi(u, 1,0)$ homogeneous we can conclude

$$
\|\Pi(u, 1,0)\|_{L^{2}\left(Q_{1}^{-}\right)} \leqslant C\left\|\tilde{D}^{2} u\right\|_{L^{2}\left(Q_{1}^{-}\right)} .
$$

The third statement now follows by a simple change of variables.

Lemma 12 Assume $H u \in L^{\infty}\left(Q_{1}^{-}\right)$. Then for every $X^{0}=\left(x^{0}, t^{0}\right) \in Q_{1 / 2}^{-}$and $r<\frac{1}{4}$, the following inequality holds

$$
\left\|\tilde{D}^{2}\left(\frac{u\left(r x+x^{0}, r^{2} t+t^{0}\right)}{r^{2}}-\Pi\left(u, r, X^{0}\right)\right)\right\|_{L^{2}\left(Q_{1}^{-}\right)} \leqslant C\left(\|u\|_{L^{1}\left(Q_{1}^{-}\right)}+\|H u\|_{L^{\infty}\left(Q_{1}^{-}\right)}\right),
$$

where $C$ depends only on the dimension. 
Proof. Recall that

$$
\tilde{D}^{2} u=\left(\begin{array}{lr}
D^{2} u & 0 \\
0 & \partial_{t} u
\end{array}\right)
$$

From Theorem 5 it follows that

$$
\left\|\tilde{D}^{2} u-\left(\tilde{D}^{2} u\right)_{r, X^{0}}\right\|_{L^{2}\left(Q_{r}^{-}\left(X^{0}\right)\right)} \leqslant C\left(\|u\|_{L^{1}\left(Q_{1}^{-}\right)}+\|H u\|_{L^{\infty}}\left(Q_{1}^{-}\right)\right) r^{(n+2) / 2} .
$$

We also observe that

$$
\begin{aligned}
& \quad f_{Q_{r}^{-}\left(X^{0}\right)} \tilde{D}^{2} u=f_{Q_{r}^{-}\left(X^{0}\right)}\left(\left(\tilde{D}^{2} u-\frac{H u}{n+1} I\right)+\frac{H u}{n+1} I\right) \\
& =M\left(X^{0}, r\right)+f_{Q_{r}^{-}\left(X^{0}\right)} \frac{H u}{n+1} I,
\end{aligned}
$$

where $M\left(X^{0}, r\right)$ is a constant matrix with zero trace and $I$ the identity matrix. Thus, if

$$
q_{X^{0}, r}=\frac{1}{2} x^{T}\left[M\left(X^{0}, r\right)\right]_{n \times n} x+t\left[M\left(X^{0}, r\right)\right]_{n+1, n+1},
$$

then

$$
\tilde{D}^{2} q_{X^{0}, r}=M\left(X^{0}, r\right)
$$

It follows that

$$
\left(\tilde{D}^{2} u\right)_{r, X^{0}}=f_{Q_{r}^{-}\left(X^{0}\right)} \tilde{D}^{2} u=\tilde{D}^{2} q_{X^{0}, r}+f_{Q_{r}^{-}\left(X^{0}\right)} \frac{H u}{n+1} I
$$

Hence,

$$
\begin{gathered}
\left\|\tilde{D}^{2} u-\tilde{D}^{2} q_{X^{0}, r}\right\|_{L^{2}\left(Q_{r}^{-}\left(X^{0}\right)\right)} \leqslant \\
\leqslant\left\|\tilde{D}^{2} u-\int_{Q_{r}^{-}\left(X^{0}\right)} \tilde{D}^{2} u\right\|_{L^{2}\left(Q_{r}^{-}\left(X^{0}\right)\right)}+\left\|\frac{H u}{n+1} I\right\|_{L^{2}\left(Q_{r}^{-}\left(X^{0}\right)\right)} .
\end{gathered}
$$

From this, the definition of $\Pi$ and rescaling the inequality (13), the lemma follows.

\section{The key proposition}

In this section we present Proposition 14, which is the base of the whole paper. First a lemma.

Lemma 13 Assume that $v$ satisfies (9) and that $w$ solves

$$
\begin{cases}H w(x, t)=f\left(r x, r^{2} t\right) & \text { in } Q_{1}^{-}, \\ w(x, t)=u_{r}(x, t)-\Pi(u, r, 0) & \text { on } \partial_{p} Q_{1}^{-},\end{cases}
$$

where

$$
u_{r}(x, t)=\frac{u\left(r x, r^{2} t\right)}{r^{2}}
$$

Then

$$
\left\|\tilde{D}^{2} w\right\|_{L^{\infty}\left(Q_{1 / 2}^{-}\right)} \leqslant C\left(\|u\|_{L^{1}\left(Q_{1}^{-}\right)}+\left\|\tilde{D}^{2} v\right\|_{L^{\infty}\left(Q_{1}^{-}\right)}\right) .
$$


Proof. To simplify the notation write $S_{r}=S(u, r, 0)$ and $p_{r}=p_{u, r, 0}$. Define the function

$$
\tilde{u}=u_{r}-S_{r} p_{r}-\left(u_{r}-S_{r} p_{r}\right) Q_{1}^{-}-x \cdot\left(\nabla\left(u_{r}-S_{r} p_{r}\right) Q_{1}^{-}\right) .
$$

Since Lemma 12 implies

$$
\left\|\tilde{D}^{2}\left(u_{r}-S_{r} p_{r}\right)\right\|_{L^{2}\left(Q_{1}^{-}\right)} \leqslant C\left(\|u\|_{L^{1}\left(Q_{1}^{-}\right)}+\|H u\|_{L^{\infty}\left(Q_{1}^{-}\right)}\right)
$$

we can use Lemma 9 to obtain

$$
\|\tilde{u}\|_{L^{2}\left(Q_{\frac{7}{8}}^{-}\right)} \leqslant C\left(\|u\|_{L^{1}\left(Q_{1}^{-}\right)}+\|H u\|_{L^{\infty}\left(Q_{1}^{-}\right)}\right) .
$$

Now we observe that $H \tilde{u}=H u$ so that interior estimates (Lemma 8) combined with (14) imply

$$
\begin{aligned}
\left|\left(u_{r}-S_{r} p_{r}\right) Q_{1}^{-}\right|+\left|x \cdot\left(\nabla\left(u_{r}-S_{r} p_{r}\right)\right)_{Q_{1}^{-}}\right|=\left|\tilde{u}_{r}(0)\right| & +\left|\nabla \tilde{u}_{r}(0)\right| \leqslant \\
& \leqslant C\left(\|u\|_{L^{1}\left(Q_{1}^{-}\right)}+\|H u\|_{L^{\infty}\left(Q_{1}^{-}\right)}\right) .
\end{aligned}
$$

As a consequence

$$
\left\|u-S_{r} p_{r}\right\|_{L^{2}\left(Q_{1}^{-}\right)} \leqslant C\left(\|u\|_{L^{1}\left(Q_{1}^{-}\right)}+\|H u\|_{\left.L^{\infty}\left(Q_{1}^{-}\right)\right)} .\right.
$$

To obtain the desired estimate, we apply interior estimates (Lemma 8) to $w$ together with the definition of $v$.

We recall the notation

$$
\lambda_{r}=\frac{\left|\Lambda \cap Q_{r}^{-}\right|}{\left|Q_{r}^{-}\right|} .
$$

Proposition 14 Let $u$ be a solution of (1) and let $v$ satisfy (9). Then there exist $C_{0}$ and $C_{1}$ depending only on the dimension such that if $X^{0} \in \Lambda \cap Q_{1 / 2}^{-}$and $r<\frac{1}{4}$ then

$$
\frac{C_{0}\left\|\tilde{D}^{2} v\right\|_{L^{\infty}\left(Q_{1}^{-}\right)}}{S\left(r, u, X^{0}\right)-C_{1}\left(\|u\|_{L^{1}\left(Q_{1}^{-}\right)}+\left\|\tilde{D}^{2} v\right\|_{L^{\infty}\left(Q_{1}^{-}\right)}\right)} \lambda_{r}^{1 / 2} \geqslant \lambda_{\frac{r}{2}}^{1 / 2},
$$

whenever

$$
S\left(r, u, X^{0}\right)>2 C_{1}\left(\|u\|_{L^{1}\left(B_{1}\right)}+\left\|\tilde{D}^{2} v\right\|_{L^{\infty}\left(B_{1}\right)}\right) .
$$

Proof. For simplicity let $X^{0}=0$ and

$$
u_{r}(X)=w_{r}(X)+S(u, r, 0) p_{u, r, 0}(X)+g_{r}(X),
$$

where

$$
\begin{cases}H g_{r}(x, t)=-f\left(r x, r^{2} t\right) \chi_{\Lambda\left(u\left(r x, r^{2} t\right)\right)} & \text { in } Q_{1}^{-}, \\ g_{r}=0 & \text { on } \partial_{p} Q_{1}^{-},\end{cases}
$$

and

$$
\begin{cases}H w_{r}(x, t)=f\left(r x, r^{2} t\right) & \text { in } Q_{1}^{-}, \\ w_{r}(x, t)=u_{r}(x, t)-S(u, r, 0) p_{u, r, 0}(X) & \text { on } \partial_{p} Q_{1}^{-}\end{cases}
$$


From Lemma 7 and Lemma 13 it follows that

$$
\left\|\tilde{D}^{2} g_{r}\right\|_{L^{2}\left(Q_{1 / 2}^{-}\right)} \leqslant C\|f\|_{L^{\infty}}\left\|\chi_{\Lambda\left(u\left(r x, r^{2} t\right)\right)}\right\|_{L^{2}\left(Q_{1}^{-}\right)},
$$

and

$$
\left\|\tilde{D}^{2} w_{r}\right\|_{L^{\infty}\left(Q_{1 / 2}^{-}\right)} \leqslant C\left(\|u\|_{L^{1}\left(Q_{1}^{-}\right)}+\left\|\tilde{D}^{2} v\right\|_{L^{\infty}\left(Q_{1}^{-}\right)}\right) .
$$

At this stage we use that $D^{2} u=\partial_{t} u=0$ a.e. in $\{u=0\}$ and thus

$$
0=\left\|\tilde{D}^{2} u_{r}\right\|_{L^{2}\left(\Lambda_{r} \cap Q_{\frac{1}{2}}^{-}\right)}=\left\|\tilde{D}^{2}\left(w_{r}+S(u, r, 0) p_{u, r, 0}+g_{r}\right)\right\|_{L^{2}\left(\Lambda_{r} \cap Q_{\frac{1}{2}}^{-}\right)},
$$

which implies

$$
\left\|\tilde{D}^{2} S(u, r, 0) p_{u, r, 0}\right\|_{L^{2}\left(\Lambda_{r} \cap Q_{\frac{1}{2}}^{-}\right)} \leqslant\left\|\tilde{D}^{2} w_{r}\right\|_{L^{2}\left(\Lambda_{r} \cap Q_{\frac{1}{2}}^{-}\right)}+\left\|\tilde{D}^{2} g_{r}\right\|_{L^{2}\left(\Lambda_{r} \cap Q_{\frac{1}{2}}^{-}\right)} .
$$

From the definition of $p_{u, r, 0}$ it follows that

$$
\left\|\tilde{D}^{2} p_{u, r, 0}\right\|_{L^{2}\left(\Lambda_{r} \cap Q_{\frac{1}{2}}^{-}\right)} \geqslant\left\|\tilde{D}^{2} p_{u, r, 0}\right\|_{L^{\infty}\left(\Lambda_{r} \cap Q_{\frac{1}{2}}^{-}\right.} \lambda_{r}^{\frac{1}{2}} \geqslant c \lambda_{\frac{r}{2}}^{\frac{1}{2}} .
$$

In addition, the estimates in (15) and (16) imply

$$
\left\|\tilde{D}^{2} g_{r}\right\|_{L^{2}\left(\Lambda_{r} \cap Q_{\frac{1}{2}}^{-}\right)} \leqslant C\|f\|_{L^{\infty}\left(\Lambda_{r} \cap Q_{1}^{-}\right)} \lambda_{r}^{\frac{1}{2}} \leqslant C\left\|\tilde{D}^{2} v\right\|_{L^{\infty}\left(Q_{1}^{-}\right)} \lambda_{r}^{\frac{1}{2}} .
$$

and

$$
\left\|\tilde{D}^{2} w_{r}\right\|_{L^{2}\left(\Lambda_{r} \cap Q_{\frac{1}{2}}^{-}\right)} \leqslant C\left(\|u\|_{L^{1}\left(Q_{1}^{-}\right)}+\left\|\tilde{D}^{2} v\right\|_{L^{\infty}\left(Q_{1}^{-}\right)}\right) \lambda_{\frac{r}{2}}^{\frac{1}{2}} .
$$

Combining (18), (19) and (20) we can conclude

$$
S(u, r, 0) \lambda_{\frac{r}{2}}^{\frac{1}{2}} \leqslant C\left\|\tilde{D}^{2} v\right\|_{L^{\infty}\left(Q_{1}^{-}\right)} \lambda_{r}^{\frac{1}{2}}+C\left(\|u\|_{L^{1}\left(Q_{1}^{-}\right)}+\left\|\tilde{D}^{2} v\right\|_{L^{\infty}\left(Q_{1}^{-}\right)}\right) \lambda_{\frac{r}{2}}^{\frac{1}{2}},
$$

from which the desired result follows.

PROPOSITION 15 Let

$$
\begin{array}{ll}
H g=f(x, t) \chi_{\Lambda} & \text { in } Q_{2^{-k}}^{-} \\
\left\|\tilde{D}^{2} g\right\|_{L^{2}\left(Q_{2-k}\right)} \leqslant C_{1} 2^{-k(n+2) / 2} &
\end{array}
$$

where $\|f\|_{L^{\infty}} \leqslant C_{2}$ and

$$
\lambda_{2^{-j-1}} \leqslant \frac{1}{4} \lambda_{2^{-j}}
$$

for $j=k, k+1, k+2, \ldots, J$. Then there exists a universal constant $C_{0}$ such that

$$
\sup _{Q_{1}}\left|\Pi\left(g, 2^{-j}, 0\right)\right| \leqslant C_{0}\left(C_{1}+C_{2}\right)
$$

for $j=k, k+1, k+2, \ldots, J$. 
Proof. There is no loss of generality to assume that $k=0$. In particular, we may parabolically rescale $g$ to $\bar{g}(x, t)=g\left(2^{-k} x, 2^{-2 k} t\right) / 2^{-2 k}$. Then $\bar{g}$ satisfies the assumptions with $k=0$ and $J-k$ in place of $J$. If we can prove (22) for $\bar{g}$ then it follows for $g$ by scaling back. We may thus assume that $k=0$ in the proof.

We may write $g_{j}=\left.g\right|_{Q_{2^{-j}}^{-}}$, the restriction of $g$ to $Q_{2^{-j}}^{-}$, as the following sum

$$
g_{j}=\sum_{k=0}^{j} h_{k}+\tilde{g}_{j}
$$

where

$$
\begin{array}{ll}
H \tilde{g}_{j}=f \chi_{\Lambda} & \text { in } Q_{2^{-j}}^{-} \\
\tilde{g}_{j}=0 & \text { on } \partial_{p} Q_{2^{-j}}^{-}
\end{array}
$$

and

$$
\begin{array}{ll}
H h_{k}=0 & \text { in } Q_{2^{-k}}^{-} \\
h_{k}=\tilde{g}_{k-1} & \text { on } \partial_{p} Q_{2^{-k}}^{-},
\end{array}
$$

where we, for consistency, identify $g_{-1}=g$. That $\tilde{g}_{j}=g$ in $Q_{2^{-j}}^{-}$follows by an easy induction. It is true, by definition, for $j=-1$. If $\tilde{g}_{j-1}=g$ in $Q_{2^{-j+1}}^{-}$then $H \tilde{g}_{j}=H g$ in $Q_{2^{-j}}^{-}$and $\tilde{g}_{j}=g$ on $\partial_{p} Q_{2^{-j}}^{-}$by construction. By the maximum principle it follows that $\tilde{g}_{j}=g$ in $Q_{2^{-j}}^{-}$and our induction is complete.

Next we notice that by (21) and Hölder's inequality it follows that

$$
\left\|H \tilde{g}_{j}\right\|_{L^{2}\left(Q_{2^{-j}}^{-}\right)} \leqslant C_{2} 2^{-j}\left|Q_{2^{-j}}^{-}\right|^{1 / 2} .
$$

This implies, by Lemma 7, that

$$
\left\|\tilde{g}_{j}\right\|_{W_{2}^{2,1}\left(Q_{2^{-j}}^{-}\right)} \leqslant C C_{2} 2^{-j}\left|Q_{2^{-j}}^{-}\right|^{1 / 2}
$$

for some universal constant $C$.

Since $h_{j+1}$ is caloric with $\tilde{g}_{j}$ as boundary values it follows from Lemma 7 and Lemma 11 that

$$
\sup _{Q_{1}^{-}}\left|\Pi\left(h_{j+1}, 2^{-k}, 0\right)\right| \leqslant C C_{2} 2^{-j}
$$

for some universal constant $C$ and all $k \geqslant j+1$ and all $j \geqslant 0$.

From (24) we may deduce, using Lemma 11 and a simple rescaling, that

$$
\sup _{Q_{1}^{-}}\left|\Pi\left(\tilde{g}_{j}, 2^{-j}, 0\right)\right| \leqslant C C_{2} 2^{-j} .
$$

We need to estimate the projection of $h_{0}$ as well. From parabolic estimates (cf. Lemma 7) we can conclude that

$$
\left\|\tilde{D}^{2} h_{0}\right\|_{L^{2}\left(Q_{1}^{-}\right)} \leqslant C\left\|\tilde{D}^{2} g\right\|_{L^{2}\left(Q_{1}^{-}\right)}
$$

and thus, using Lemma 11 again, that

$$
\sup _{Q_{1}^{-}}\left|\Pi\left(h_{0}, 2^{-k}, 0\right)\right| \leqslant C C_{1}
$$


for any $k \geqslant 0$.

Using the linearity of $\Pi$ we can finally conclude that for any $j \leqslant J$

$$
\begin{aligned}
\sup _{Q_{1}^{-}}\left|\Pi\left(g, 2^{-j}, 0\right)\right| & \leqslant \sum_{k=0}^{j} \sup _{Q_{1}^{-}}\left|\Pi\left(h_{k}, 2^{-j}, 0\right)\right|+\left|\Pi\left(\tilde{g}_{j}, 2^{-j}, 0\right)\right| \\
& \leqslant C\left(C_{1}+C_{2} \sum_{k=0}^{j} 2^{-k}+C_{2}\right) \leqslant C\left(C_{1}+3 C_{2}\right),
\end{aligned}
$$

where we have used (23) in the first inequality and (25), (26) and (27) in the second.

\section{Proof of the main result}

The two following lemmata provides us with the result that if $S$ is bounded then $u$ is $W_{\infty}^{2,1}$.

LEMMA 16 (Quadratic growth implies $W_{\infty}^{2,1}$ ) Suppose $v$ satisfies (9) and $u$ is a solution of (1) such that

$$
\sup _{Q_{r}^{-}\left(Y^{0}\right)}|u| \leqslant M r^{2}
$$

for all $0<r<1 / 2$ and $Y^{0} \subset Q_{\frac{1}{2}}^{-} \cap \partial\{u \neq 0\}$. Then

$$
\left\|\tilde{D}^{2} u\right\|_{L^{\infty}\left(Q_{\frac{1}{2}}^{-}\right)} \leqslant C\left(M+\|u\|_{L^{1}\left(Q_{1}^{-}\right)}+\left\|D^{2} v\right\|_{L^{\infty}\left(Q_{1}^{-}\right)}\right) .
$$

Proof. Take $X^{0} \in Q_{\frac{1}{2}}^{-}$and define

$$
r=\sup \left\{r: Q_{r}^{-}\left(X^{0}\right) \cap \partial\{u \neq 0\}=\emptyset\right\} .
$$

We now split the proof into two cases:

Case 1: $r<\frac{1}{4}$. From the definition of $r$ it follows that with

$$
w=u-v-v\left(X^{0}\right)-\nabla v\left(X_{0}\right) \cdot\left(x^{0}-x\right),
$$

then $H w=0$ in $Q_{r}^{-}\left(X^{0}\right)$. From Lemma 6

$$
\left\|\tilde{D}^{2} w\right\|_{L^{\infty}\left(Q_{r / 2}^{-}\left(X^{0}\right)\right)} \leqslant \frac{C}{r^{2}}\|u\|_{L^{\infty}\left(Q_{r}^{-}\left(X^{0}\right)\right)} \leqslant C\left(M+\left\|D^{2} v\right\|_{L^{\infty}\left(Q_{r}^{-}\left(X^{0}\right)\right)}\right),
$$

and thus

$$
\left\|\tilde{D}^{2} u\right\|_{L^{\infty}\left(Q_{r / 2}^{-}\left(X^{0}\right)\right)} \leqslant C\left(M+\left\|D^{2} v\right\|_{L^{\infty}\left(Q_{1}^{-}\right)}\right),
$$

where $C$ is possibly a larger constant.

Case 2: $r \geqslant \frac{1}{4}$. In this case, it is clear that

$$
w=u-v-v\left(X^{0}\right)-\nabla v\left(X_{0}\right) \cdot\left(x^{0}-x\right),
$$


then $H w=0$ in $Q_{\frac{1}{4}}^{-}\left(X^{0}\right)$. Lemma 6 then implies

$$
\left\|\tilde{D}^{2} w\right\|_{L^{\infty}\left(Q_{1 / 8}^{-}\left(X^{0}\right)\right)} \leqslant C\|u\|_{L^{1}\left(Q_{\frac{1}{4}}^{-}\left(X^{0}\right)\right)} \leqslant C\left(\|u\|_{L^{1}\left(Q_{1}^{-}\right)}+\left\|D^{2} v\right\|_{L^{\infty}\left(Q_{\frac{1}{4}}^{-}\left(X^{0}\right)\right)}\right),
$$

which implies

$$
\left\|\tilde{D}^{2} u\right\|_{L^{\infty}\left(Q_{1 / 8}^{-}\left(X^{0}\right)\right)} \leqslant C\left(\|u\|_{L^{1}\left(Q_{1}^{-}\right)}+\left\|D^{2} v\right\|_{L^{\infty}\left(Q_{1}^{-}\right)}\right) .
$$

The combination of the two cases above yields the desired estimate.

LEMma 17 (Bounded $S$ implies quadratic growth) Suppose $u$ is a solution of (1) and let $X^{0} \in$ $Q_{\frac{1}{2}}^{-} \cap \partial\{u \neq 0\}$. Then for $r<1 / 4$

$$
\sup _{Q_{\frac{\Gamma}{2}}^{-}\left(X^{0}\right)}|u| \leqslant C\left(S\left(u, r, X^{0}\right)+\|u\|_{L^{1}\left(Q_{1}^{-}\right)}+\|H u\|_{L^{\infty}\left(Q_{1}^{-}\right)}\right) r^{2} .
$$

The proof is very similar to the proof of Lemma 13 and therefore we give only a sketch of the proof.

Proof. To avoid cumbersome writing we use the notation $S=S\left(u, r, X^{0}\right)$ and

$$
u_{r}=u_{r, X^{0}}=\frac{u\left(r x+x^{0}, r^{2} t+t^{0}\right)}{r^{2}},
$$

where $X^{0}=\left(x^{0}, t^{0}\right)$, throughout the whole proof. The hypotheses of the lemma and Lemma 12 imply

$$
\left\|\tilde{D}^{2} u_{r}\right\|_{L^{2}\left(Q_{1}^{-}\right)} \leqslant C\left(S+\|u\|_{L^{1}\left(Q_{1}^{-}\right)}+\|H u\|_{L^{\infty}\left(Q_{1}^{-}\right)}\right) .
$$

Defining

$$
\tilde{u}=u_{r}-\left(u_{r}\right) Q_{1}^{-}-x \cdot\left(\nabla\left(u_{r}\right) Q_{1}^{-}\right),
$$

we can reason as in the proof of Lemma 13 to obtain that

$$
\begin{aligned}
\|\tilde{u}\|_{L^{\infty}\left(Q_{\frac{1}{2}}^{-}\right)}+\left|\left(u_{r}\right)_{Q_{2}^{-}}\right|+\left|x \cdot\left(\nabla\left(u_{r}\right)\right)_{Q_{2}^{-}}\right| & =\|\tilde{u}\|_{L^{\infty}\left(Q_{\frac{1}{2}}^{-}\right)}+\left|\tilde{u}_{r}(0)\right|+\left|\nabla \tilde{u}_{r}(0)\right| \\
& \leqslant C\left(S+\|u\|_{L^{1}\left(Q_{1}^{-}\right)}+\|H u\|_{L^{\infty}\left(Q_{1}^{-}\right)}\right) .
\end{aligned}
$$

This implies, by the triangle inequality

$$
\left\|u_{r}\right\|_{L^{\infty}\left(Q_{\frac{1}{2}}^{-}\right)} \leqslant C\left(S+\|u\|_{L^{1}\left(Q_{1}^{-}\right)}+\|H u\|_{L^{\infty}\left(Q_{1}^{-}\right)}\right) .
$$

We are now ready to give the proof of the main theorem.

Proof of Theorem 1. In view of Lemma 17 and Lemma 16 it is enough to prove that

$$
\sup _{Q_{1}^{-}}\left|\Pi\left(u, 2^{-j}, X^{0}\right)\right| \leqslant C\left(\|u\|_{L^{1}\left(Q_{1}^{-}\right)}+\left\|D^{2} v\right\|_{L^{\infty}\left(Q_{1}^{-}\right)}\right)
$$


for $j=2,3,4, \ldots$ and some universal constant $C$ and every $X^{0} \in Q_{1 / 2}^{-}$. It is enough to prove (28) for $X^{0}=0$. Once (28) is proved for $X^{0}=0$ a translation argument assures that (28) holds for any $X^{0} \in Q_{1 / 2}^{-}$. Then Lemma 17 provides a quadratic bound on the solution which, by Lemma 16 implies regularity.

Let us denote by $B$ the set $B \subset \mathbb{N}$ of all $j \in \mathbb{N}$ such that

$$
S\left(u, 2^{-j}, 0\right) \geqslant 2 C_{0}\left\|\tilde{D}^{2} v\right\|_{L^{\infty}\left(Q_{1}^{-}\right)}+2 C_{1}\left(\|u\|_{L^{1}\left(Q_{1}^{-}\right)}+\left\|\tilde{D}^{2} v\right\|_{L^{\infty}\left(Q_{1}^{-}\right)}\right),
$$

where $C_{0}$ and $C_{1}$ are as is Proposition 14 . Naturally if $j \notin B$ then

$$
S\left(u, 2^{-j}, 0\right) \leqslant 2 C_{0}\left\|\tilde{D}^{2} v\right\|_{L^{\infty}\left(Q_{1}^{-}\right)}+2 C_{1}\left(\|u\|_{L^{1}\left(Q_{1}^{-}\right)}+\left\|\tilde{D}^{2} v\right\|_{L^{\infty}\left(Q_{1}^{-}\right)}\right)
$$

for all $j$ which in turn implies (28) with $C=2\left(c_{0}+C_{1}\right)$. Therefore $B$ consists of the "bad" scales where (28) might not hold.

If $j \in B$ then, according to Proposition 14,

$$
\frac{1}{4} \lambda_{2^{-j}} \geqslant \lambda_{2^{-j-1}}
$$

Moreover, if $\{k, k+1, k+2, \ldots, J\} \subset B$ then (29) holds for all $j \in\{k, k+1, \ldots, J\}$. In particular, if $\{k, k+1, k+2, \ldots, J\} \subset B$ and if we split $u$ into

$$
u=\tilde{v}+g
$$

where $H \tilde{v}=H v$ and $H g=-f \chi_{\Lambda}$ then $g$ satisfies the conditions in Proposition 15 for $j \in\{k, k+$ $1, \ldots, J\}$. We have a choice in $h$ and $g$ and we may choose $g$ such that $\|g\|_{L^{1}\left(Q_{1}^{-}\right)} \leqslant C\|f\|_{L^{\infty}\left(Q_{1}^{-}\right)}$ by for instance letting $g$ be the convolution of the heat kernel and $-f \chi_{\Lambda}$. Then Theorem 5 implies that

$$
\|\tilde{D} g\|_{B M O\left(Q_{1 / 2}^{-}\right)} \leqslant C\|f\|_{L^{\infty}\left(Q_{1}^{-}\right)} .
$$

Then it follows, by the triangle inequality, that

$$
\|\tilde{v}\|_{L^{1}\left(Q_{1}^{-}\right)} \leqslant\|u\|_{L^{1}\left(Q_{1}^{-}\right)}+C\|f\|_{L^{\infty}\left(Q_{1}^{-}\right)} .
$$

Let $j \in \mathbb{N}$. Then either $j \notin B$ and (28) holds or $j \in B$ and there exists a smallest $k \in \mathbb{N}$ such that $\{k, k+1, k+2, \ldots, j\} \subset B$. Using (30), the linearity of $\Pi$ and the triangle inequality we can estimate

$$
\begin{aligned}
& \sup _{Q_{1}^{-}}\left|\Pi\left(u, 2^{-j}, 0\right)-\Pi\left(u, 2^{-k}, 0\right)\right| \\
& \quad \leqslant \sup _{Q_{1}^{-}}\left|\Pi\left(\tilde{v}, 2^{-j}, 0\right)-\Pi\left(\tilde{v}, 2^{-k}, 0\right)\right|+\sup _{Q_{1}^{-}}\left|\Pi\left(g, 2^{-j}, 0\right)-\Pi\left(g, 2^{-k}, 0\right)\right| .
\end{aligned}
$$

Since $v \in W_{\infty}^{2,1}\left(Q_{1}^{-}\right)$it follows from Lemma 6 and Lemma 11 that

$$
\begin{aligned}
\sup _{Q_{1}^{-}}\left|\Pi\left(\tilde{v}, 2^{-j}, 0\right)\right|, \sup _{Q_{1}^{-}}\left|\Pi\left(\tilde{v}, 2^{-k}, 0\right)\right| & \leqslant C\left(\left\|\tilde{D}^{2} v\right\|_{L^{\infty}\left(Q_{1}^{-}\right)}+\|\tilde{v}\|_{L^{1}\left(Q_{1}^{-}\right)}\right) \\
& \leqslant C\left(\left\|\tilde{D}^{2} v\right\|_{L^{\infty}\left(Q_{1}^{-}\right)}+\|u\|_{L^{1}\left(Q_{1}^{-}\right)}\right),
\end{aligned}
$$


where we used (32) in the last inequality.

We use Proposition 15 to estimate

$$
\sup _{Q_{1}^{-}}\left|\Pi\left(g, 2^{-j}, 0\right)-\Pi\left(g, 2^{-k}, 0\right)\right|=\sup _{Q_{1}^{-}}\left|\Pi\left(\tilde{g}, 2^{-j}, 0\right)\right|
$$

where $\tilde{g}=g-\Pi\left(g, 2^{-k}, 0\right)$. In particular, by (31) and Lemma 12 , it follows that

$$
\|\tilde{g}\|_{L^{2}\left(Q_{2^{-k}}^{-}\right)} \leqslant C\|f\|_{L^{\infty}\left(Q_{1}^{-}\right) 2^{-k(n+2) / 2} .}
$$

Moreover, by (29) and our assumption that $\{k, k+1, \ldots, j\} \subset B$ the assumptions in Proposition 15 are satisfied with $C_{1}=C\|f\|_{L^{\infty}\left(Q_{1}^{-}\right)}$for some universal constant $C$. From Proposition 15 and (35) it therefore follows that

$$
\sup _{Q_{1}^{-}}\left|\Pi\left(g, 2^{-j}, 0\right)-\Pi\left(g, 2^{-k}, 0\right)\right|=\sup _{Q_{1}^{-}}\left|\Pi\left(\tilde{g}, 2^{-j}, 0\right)\right| \leqslant C\|f\|_{L^{\infty}\left(Q_{1}^{-}\right)} .
$$

From (33), (34) and (36) we can conclude that

$$
\sup _{Q_{1}^{-}}\left|\Pi\left(u, 2^{-j}, 0\right)\right| \leqslant \sup _{Q_{1}^{-}}\left|\Pi\left(u, 2^{-k}, 0\right)\right|+C\left(\left\|\tilde{D}^{2} v\right\|_{L^{\infty}\left(Q_{1}^{-}\right)}+\|u\|_{L^{1}\left(Q_{1}^{-}\right)}\right) .
$$

We need to estimate $\sup _{Q_{1}^{-}}\left|\Pi\left(u, 2^{-k}, 0\right)\right|$. Remember that $k \in \mathbb{N}$ was the smallest constant such that $\{k, k+1, \ldots, j\} \subset B$. This implies that $k-1 \notin B$ so

$$
S\left(u, 2^{-k+1}, 0\right) \leqslant 2 C_{0}\left\|\tilde{D}^{2} v\right\|_{L^{\infty}\left(Q_{1}^{-}\right)}+2 C_{1}\left(\|u\|_{L^{1}\left(Q_{1}^{-}\right)}+\left\|\tilde{D}^{2} v\right\|_{L^{\infty}\left(Q_{1}^{-}\right)}\right) .
$$

Furthermore, by Lemma 12,

$$
\left\|\tilde{D}^{2}\left(u-\Pi\left(u, 2^{-k+1}, 0\right)\right)\right\|_{L^{2}\left(Q_{2^{-k+1}}^{-}\right)} \leqslant C 2^{-(n+2)(k-1) / 2}\left(\|f\|_{L^{\infty}\left(Q_{1}^{-}\right)}+\|u\|_{L^{1}\left(Q_{1}^{-}\right)}\right) .
$$

That is

$$
\begin{aligned}
\sup _{Q_{1}^{-}} \mid \Pi\left(u-\Pi\left(u, 2^{-k+1}, 0\right)\right. & \left., 2^{-k}, 0\right) \mid \\
& \leqslant C\left\|\Pi\left(u-\Pi\left(u, 2^{-k+1}, 0\right), 2^{-k}, 0\right)\right\|_{L^{2}\left(Q_{1}^{-}\right)} \\
& \leqslant 2^{-k-k(n+2) / 2} C\left\|\Pi\left(u-\Pi\left(u, 2^{-k+1}, 0\right), 2^{-k}, 0\right)\right\|_{L^{2}\left(Q_{2^{-k}}^{-}\right)} \\
& \leqslant 2^{-(n+2) k / 2} C\left\|\tilde{D}^{2}\left(u-\Pi\left(u, 2^{-k+1}, 0\right)\right)\right\|_{L^{2}\left(Q_{2^{-k}}^{-}\right)} \\
& \leqslant C\left(\|f\|_{L^{\infty}\left(Q_{1}^{-}\right)}+\|u\|_{L^{1}\left(Q_{1}^{-}\right)}\right)
\end{aligned}
$$

where we used that $\Pi(\cdot)$ is a polynomial in the first inequality, a rescaling in the second, Lemma 11 in the third and Lemma 12 in the last inequality. The triangle inequality, (38) and (39) implies that

$$
\begin{aligned}
\sup _{Q_{1}^{-}}\left|\Pi\left(u, 2^{-k}, 0\right)\right| & \leqslant C 2^{(n+2) / 2}\left(\|f\|_{L^{\infty}\left(Q_{1}^{-}\right)}+\|u\|_{L^{1}\left(Q_{1}^{-}\right)}\right)+\sup _{Q_{1}^{-}}\left|\Pi\left(u, 2^{-k+1}, 0\right)\right| \\
& \leqslant C\left(\|f\|_{L^{\infty}\left(Q_{1}^{-}\right)}+\|u\|_{L^{1}\left(Q_{1}^{-}\right)}\right) .
\end{aligned}
$$


Using (40) in (37) yields

$$
\sup _{Q_{1}^{-}}\left|\Pi\left(u, 2^{-j}, 0\right)\right| \leqslant C\left(\left\|\tilde{D}^{2} v\right\|_{L^{\infty}\left(Q_{1}^{-}\right)}+\|u\|_{L^{1}\left(Q_{1}^{-}\right)}\right),
$$

where we also used that $\|f\|_{L^{\infty}} \leqslant C\left\|\tilde{D}^{2} v\right\|_{L^{\infty}}$. Equation (41) is valid for all $j \in \mathbb{N}$.

By translating the coordinate system it follows from (41) that

$$
\sup _{Q_{1}^{-}}\left|\Pi\left(u, 2^{-j}, X^{0}\right)\right| \leqslant C\left(\left\|\tilde{D}^{2} v\right\|_{L^{\infty}\left(Q_{1}^{-}\right)}+\|u\|_{L^{1}\left(Q_{1}^{-}\right)}\right)
$$

for any $X^{0} \in Q_{1 / 2}^{-}$.

Lemma 17 and (42) implies, for all $r \in(0,1 / 4)$, that

$$
\sup _{Q_{r}^{-}\left(X^{0}\right)}|u| \leqslant C\left(\left\|\tilde{D}^{2} v\right\|_{L^{\infty}\left(Q_{1}^{-}\right)}+\|u\|_{L^{1}\left(Q_{1}^{-}\right)}\right) .
$$

which by Lemma 16 implies that

$$
\left\|\tilde{D}^{2} u\right\|_{L^{\infty}\left(Q_{1 / 2}^{-}\right)} \leqslant C\left(\left\|\tilde{D}^{2} v\right\|_{L^{\infty}\left(Q_{1}^{-}\right)}+\|u\|_{L^{1}\left(Q_{1}^{-}\right)}\right),
$$

and the proof is complete.

\section{Regularity of the free boundary}

In this section we prove the second main theorem. The idea is to prove that at low energy points (see Definition 19 below) points and at a scale small enough, the solution is non-negative.

\subsection{Weiss' monotonicity formula}

In order to prove the regularity of the free boundary we need to introduce some notions from for instance [4].

Define the Weiss energy for $v(x, t): \mathbb{R}^{n} \times \mathbb{R}^{-} \rightarrow \mathbb{R}$ to be

$$
W\left(r ; v, f,\left(x^{0}, t^{0}\right)\right)=\frac{1}{r^{4}} \int_{\mathbb{R}^{n} \times\left(-r^{2}+t^{0}, t^{0}\right]}\left(|\nabla v|^{2}+2 f v+\frac{v^{2}}{t}\right) G(x,-t) d x d t,
$$

and let

$$
v_{r,\left(x^{0}, t^{0}\right)}(x, t)=\frac{v\left(r x+x^{0}, r^{2} t+t^{0}\right)}{r^{2}},
$$

so that

$$
W\left(r ; v, f,\left(x^{0}, t^{0}\right)\right)=W\left(1 ; v_{r,\left(x^{0}, t^{0}\right)}, f_{r,\left(x^{0}, t^{0}\right)}, 0\right),
$$

where

$$
f_{r,\left(x^{0}, t^{0}\right)}(x, t)=f\left(r x+x^{0}, r^{2} t+t^{0}\right) .
$$

Moreover, for a general function $u(x, t)$ define

$$
L u=x \cdot \nabla u+2 t u_{t}-2 u .
$$

The following proposition is a parabolic version of Weiss' monotonicity formula. Once we know that $u$ enjoys the optimal regularity (Theorem 1), it can be proved in the same manner as in [7] or [12]. 
Proposition 18 Let $u$ be a solution of $(1), \psi \in C_{0}^{\infty}\left(B_{3 / 4}\right)$ such that $\psi=1$ on $B_{1 / 2}$, and set $v=u \psi$. Then there is a continuous function

$$
F=F\left(\|f\|_{C^{\text {Dini }}},\|u\|_{L^{1}\left(Q_{1}^{-}\right)}, r\right),
$$

with $F(0)=0$ such that

$$
W\left(r ; v, f,\left(x^{0}, t^{0}\right)\right)+F(r)
$$

is a non-decreasing function for $0<r<1 / 2$, and in particular for $0<s<r<1 / 2$ there holds

$$
\begin{array}{r}
W\left(r ; v, f,\left(x^{0}, t^{0}\right)\right)-W\left(s ; v, f,\left(x^{0}, t^{0}\right)\right)+F(r)-F(s) \\
\geqslant \int_{s}^{r} \frac{1}{\tau^{5}} \int_{\mathbb{R}^{n} \times\left(-\tau^{2}+t^{0}, t^{0}\right]} \frac{(L v)^{2}}{-t} G(x,-t) d t d x d \tau .
\end{array}
$$

In view of the proposition above, the limit

$$
W\left(0^{+} ; u, 1\right):=\lim _{r \rightarrow 0} W(r ; u, 1)=\lim _{r \rightarrow 0} W(r ; u, f)
$$

exists. In Lemma 6.2 and Lemma 6.3 in [4], the possible values are determined. It turns out that they are in general $0,15 / 2$ and 15 . As is explained in Section 7.1 in [4] the value 0 , corresponding to so-called zero energy points, does not occur for $X^{0} \in \Gamma$.

Definition 19 We say that the the point $\left(x^{0}, t^{0}\right) \in \Gamma$ is a low energy point if

$$
W\left(0^{+} ; u, f,\left(x^{0}, t^{0}\right)\right):=\lim _{r \rightarrow 0} W\left(r ; v, f,\left(x^{0}, t^{0}\right)\right)=\frac{15}{2} .
$$

From Proposition 18 it follows that the function

$$
(x, t) \mapsto W\left(0^{+} ; u, f,(x, t)\right),
$$

is upper semi-continuous, and thus the set of regular points is an open set.

\subsection{The proof}

We can now give the proof of the second main theorem.

Proof of Theorem 2. The proof consists of three steps. The first step amounts to prove that if the origin is a low energy point, then $u$ is non-negative close to the origin. The second step consists of applying the theory known for the case when $u$ has a sign, implying that the free boundary is locally a $C^{1}$ graph. These two steps prove the first part of the theorem.

In the third and final step, we observe that if the geometric condition holds at the origin, then Lemma 13.3 in [4] implies that the energy is sufficiently low (below the threshold 15) in a uniform neighbourhood of the origin. In particular, this implies that all points in that neighbourhood are low energy points. Hence, the first part of the theorem is applicable in a uniform neighborhood of the origin, which implies the second part of the theorem.

Step 1: For $r_{0}$ small enough, $u \geqslant 0$ in $Q_{r^{0}}^{-}$. We argue by contradiction. If this is not true, then there is a solution $u$ of (1), with

$$
\|u\|_{L^{1}\left(Q_{1}^{-}\right)}+\|f\|_{C^{\text {Dini }}} \leqslant M, \quad W(0 ; u, f,(0,0))=\frac{15}{2},
$$


and points $\left(y^{j}, s^{j}\right) \in Q_{1}^{-} \rightarrow(0,0)$ so that $u\left(y^{j}, s^{j}\right)<0$. Let $\left(x^{j}, t^{j}\right)$ be the closest free boundary point, i.e.,

$$
r_{j}=\operatorname{dist}\left(\Gamma,\left(y^{j}, s^{j}\right)\right)=\operatorname{dist}\left(y^{j}-x^{j}, s^{j}-t^{j}\right)=\sqrt{\left|x^{j}-y^{j}\right|^{2}+\left|t^{j}-s^{j}\right|} .
$$

Define the rescaled functions

$$
v_{j}(x, t)=\frac{u\left(r_{j} x+x^{j}, r_{j}^{2} t+t^{j}\right)}{r_{j}^{2}} .
$$

Clearly $v_{j}$ satisfies the equation

$$
H v_{j}=f\left(r_{j} x+x^{j}, r_{j}^{2} t+t^{j}\right) \chi_{\left\{v_{j} \neq 0\right\}}, \text { in } Q_{\frac{1}{r_{j}}}^{-}\left(-x^{j},-t^{j}\right) .
$$

Moreover, due to Theorem 1

$$
\sup _{Q_{\rho}^{-}}\left|v_{j}\right| \leqslant C \rho^{2}, \text { for } \rho<\frac{1}{2 r_{j}},
$$

and by the choice of $\left(y^{j}, s^{j}\right)$ and $\left(x^{j}, t^{j}\right), H v_{j}=f\left(r_{j} x+x^{j}, r_{j}^{2} t+t^{j}\right)$ in the set

$$
\left\{(x, t):\left|x-\frac{x^{j}-y^{j}}{r_{j}}\right|^{2}+\left|t-\frac{t^{j}-s^{j}}{r_{j}}\right|<1\right\} .
$$

By standard estimates for parabolic equations, we can extract a sub-sequence, again labelled $v_{j}$, such that $v_{j} \rightarrow v_{0}$ uniformly and

$$
\begin{aligned}
H v_{0}= & \chi_{\left\{v_{0} \neq 0\right\}}, \text { in } \mathbb{R}^{n} \times \mathbb{R}^{-}, \quad(0,0) \in \Gamma\left(v_{0}\right) \\
& \sup _{Q_{\rho}^{-}}\left|v_{0}\right| \leqslant C \rho^{2}, \text { for all } \rho>0,
\end{aligned}
$$

and

$$
H v_{0}=1 \text { in }\left\{(x, t):\left|x-z^{0}\right|^{2}+\left|t-\tau^{0}\right|<1\right\}, \quad v_{0}\left(z^{0}, \tau^{0}\right) \leqslant 0,
$$

where

$$
\left(z^{0}, \tau^{0}\right)=\lim _{j \rightarrow \infty}\left(\frac{y^{j}-x^{j}}{r_{j}}, \frac{s_{j}-t_{j}}{r_{j}}\right), \quad\left(z^{0}\right)^{2}+\tau^{0}=1 .
$$

Observe that (44) assures that $v_{0} \not \equiv 0$. Moreover non-degeneracy (Lemma 5.1 in [4]) implies that the origin is contained in the free boundary of $v_{0}$ (see also section 5.2 in [4]).

Next we need to use the assumption on the energy functional $W(r, u, f, X)$. Since $W(r, u, f, X)$ is uniformly continuous in $X$ for each $r>0$ and

$$
\lim _{r \rightarrow 0} W(r, u, f, 0)=\frac{15}{2},
$$

the monotonicity formula implies that for each $\varepsilon>0$ there exists an $r_{\varepsilon}>0$ such that

$$
W\left(r, u, f,\left(x^{j}, t^{j}\right)\right) \leqslant W(r, u, f, 0)+\varepsilon<\frac{15}{2}+2 \varepsilon
$$


if $r<r_{\varepsilon}$ and $j$ is large enough ( $j$ may depend on $r$ ). In particular, a rescaling implies that

$$
W\left(\frac{r}{r_{j}}, v_{j}, f\left(r_{j} x, r_{j}^{2} t\right), 0\right) \leqslant \frac{15}{2}+2 \varepsilon
$$

if $r<r_{\varepsilon}$ is small enough and $j$ large enough. Passing to the limit $j \rightarrow \infty$ and using that $W$ is almost monotone in its first argument we may conclude that for any $\varepsilon>0$

$$
W\left(r, v_{0}, 1,0\right) \leqslant \frac{15}{2}+2 \varepsilon .
$$

From (45) and the second part of Lemma 9.2 in [4] we can conclude that

$$
v_{0}=\frac{1}{2}(x \cdot e)_{+}^{2},
$$

for some unit vector $e$. This is a contradiction to (44) since $\left(z^{0}, \tau^{0}\right)$ is, by construction, a point in the set $H v_{0}=1$ at unit distance from the free boundary so by (46) $v_{0}\left(z^{0}, \tau^{0}\right)=\frac{1}{2}$ which contradicts (44).

Step 2: Apply the results from [11]. Now we are in the situation of Theorem 1.9 in [11] if we consider $u$ to be defined only in $Q_{r_{0}}^{-}$, i.e., $u \geqslant 0$ in $Q_{r_{0}}^{-}$and the origin is a low energy point. Hence, there is a small neighbourhood, which might depend on $u$ itself, where the free boundary is a $C^{1}$ graph (in the parabolic sense).

Step 3: The geometric condition implies a uniform energy condition. Theorem 1 applied to $u$ and then Lemma 13.3 in [4] applied to $u\left(r x, r^{2} t\right) / r^{2}$ implies that if $\sigma(r)$ is large enough, $r$ small enough (both depending on $\|u\|_{L^{1}\left(Q_{1}^{-}\right)}$and $\|f\|_{C^{\text {Dini }}\left(Q_{1}^{-}\right)}$) and

$$
\frac{\operatorname{MD}\left(\left\{x: u\left(x,-r^{2}\right)=0\right\} \cap B_{r}\right)}{r}>\sigma(r),
$$

then

$$
W(\rho ; u, f, X)<15-\varepsilon_{0},
$$

for all $X \in Q_{r / 2}^{-}, \rho<r$ small enough (depending on $\|u\|_{L^{1}\left(Q_{1}^{-}\right)}$and $\|f\|_{C^{\text {Dini }}\left(Q_{1}^{-}\right)}$). In particular, all free boundary points in $Q_{r / 2}^{-}$are low energy points. Hence, we can apply Step 1 and Step 2 to conclude that in $Q_{r / 2}^{-}$, the free boundary is a $C^{1}$ graph.

Acknowledgements. Both John Andersson and Erik Lindgren are grateful to the the Göran Gustafsson foundation. Erik Lindgren also thanks the Royal Swedish Academy of Sciences for partial financial support.

\section{REFERENCES}

1. Andersson, J., Lindgren, E., \& Shahgholian, H., Optimal regularity for the no-sign obstacle problem. Comm. Pure Appl. Math. 66, (2013), 245-262. Zbl06132672 MR2999297

2. Andersson, J., Shahgholian, H., \& Weiss, G. S., Uniform regularity close to cross singularities in an unstable free boundary problem. Comm. Math. Phys. 296 (2010), 251-270. Zbl1197. 35088 MR2606634 
3. Blank, I., Sharp results for the regularity and stability of the free boundary in the obstacle problem. Indiana Univ. Math. J. 50 (2001), 1077-1112. Zbl1032.35170 MR1871348

4. Caffarelli, L., Petrosyan, A., \& Shahgholian, H., Regularity of a free boundary in parabolic potential theory. J. Amer. Math. Soc. 174 (2004), 827-869 (electronic). Zbl1054. 35142 MR2083469

5. Caffarelli, L. A., A monotonicity formula for heat functions in disjoint domains. In Boundary value problems for partial differential equations and applications, vol. 29 of RMA Res. Notes Appl. Math. Masson, Paris, 1993, 53-60. Zb10808.35042 MR1260438

6. Campanato, S., Equazioni paraboliche del secondo ordine e spazi $L^{2, \theta}(\Omega, \delta)$. Ann. Mat. Pura Appl. (4) 73 (1966), 55-102. Zbl0144. 14101 MR0213737

7. EdQuist, A., \& Lindgren, E., Regularity of a parabolic free boundary problem with hölder continuous coefficients. Comm. Partial Differential Equations 37 (2012), 1161-1185. Zb106102457 MR2942980

8. Edquist, A., \& Petrosyan, A., A parabolic almost monotonicity formula. Math. Ann. 341 (2008), 429-454. Zbl1139.35045 MR2385663

9. Evans, L. C., Partial differential equations, second ed., vol. 19 of Graduate Studies in Mathematics. American Mathematical Society, (2010). Zb11194.35001 MR2597943

10. Lieberman, G. M., Second order parabolic differential equations. World Scientific Publishing Co. Inc., (1996). Zb10884.35001 MR1465184

11. Lindgren, E., \& Monneau, R., Pointwise estimates for the heat equation. application to the free boundary of the obstacle problem with Dini coefficients. Accepted for publication in Indiana Univ. Math. J. (2011).

12. Lindgren, E., \& Monneau, R., Pointwise regularity of the free boundary for the parabolic obstacle problem. ArXiv: 1305.7349 (2013).

13. MATIǏČUK, M. I., \& ĖĬDEL'MAN, S. D., Boundary value problems for second order parabolic and elliptic equations in Dini spaces. Dokl. Akad. Nauk SSSR 198 (1971), 533-536. Zb10227. 35049 MR0288425

14. Petrosyan, A., \& Shahgholian, H., Geometric and energetic criteria for the free boundary regularity in an obstacle-type problem. Amer. J. Math. 129 (2007), 1659-1688. Zb11133. 35108 MR2369892

15. Schaeffer, D. G., Some examples of singularities in a free boundary. Ann. Scuola Norm. Sup. Pisa Cl. Sci. (4) 4 (1977), 133-144. Zb10354. 35033 MR0516201

16. Wang, X., Schauder Estimates for Elliptic and Parabolic equations. Chinese Annals of MathematicsSeries B 27 (2006), 637-642. Zbl1151.35329 MR2273802 